\title{
The formation of close-in planets by the slingshot model
}

\author{
Makiko Nagasawa ${ }^{1}$, Shigeru Ida ${ }^{2}$ and Taisuke Bessho ${ }^{2}$ \\ ${ }^{1}$ Global Edge Institute, Tokyo Institute of Technology, \\ 2-12-1 Ookayama, Meguro-ku, Tokyo 152-8550, Japan \\ email: nagasawa.m.ad@m.titech.ac.jp \\ ${ }^{2}$ Dept. of Earth \& Planetary Sciences, Tokyo Institute of Technology \\ 2-12-1 Ookayama, Meguro-ku, Tokyo 152-8551, Japan
}

\begin{abstract}
We investigated the efficiency of planet scatterings in producing close-in planets by a direct inclusion of the dynamical tide effect into the simulations. We considered a system consists of three Jovian planets. Through a planet-planet scattering, one of the planets is sent into shorter orbit. If the eccentricity of the scattered planet is enough high, the tidal dissipation from the star makes the planetary orbit circular. We found that the short-period planets are formed at about $30 \%$ cases in our simulation and that Kozai mechanism plays an important role. In the Kozai mechanism, the high inclination obtained by planet-planet scattering is transformed to the eccentricity. It leads the pericenter of the innermost planet to approach the star close enough for tidal circularization. The formed close-in planets by this process have a widely spread inclination distribution. The degree of contribution of the process for the formation of close-in planets will be revealed by more observations of Rossiter-McLaughlin effects for transiting planets.
\end{abstract}

Keywords. planetary systems: formation, methods: numerical

\section{Introduction}

More than 260 planets have been detected around both solar and non-solar type stars. In Figure 1, we display the semi-major axis versus the eccentricity of 234 planets $\dagger$ which have been discovered by the radial velocity techniques around solar type stars. The dotted line shows pericenter distance $q=0.05$ AU. Approximately $17 \%$ of planets have been discovered inside of $q<0.05 \mathrm{AU}$. Many of these planets have circular orbits compared with other extrasolar planets and this property is accounted for by the circularization due to the tidal dissipation of energy within the planetary envelopes (Rasio \& Ford 1996).

Close-in planets are considered to be formed beyond ice line and to be moved to shorterperiod orbits later. One promising mechanism for migration is the tidal interaction with protoplanetary gas disk (e.g., Ida \& Lin 2004a, Ida \& Lin 2004b). However, while several close-in planets without nearby secondary planets have moderate eccentricities $(\sim 0.2)$, it is not clear that this mechanism alone can excite eccentricity up to that level. Alternative idea is the "Slingshot model". This is a coupled process of planet-planet interaction ("Jumping Jupiters model" (Rasio \& Ford 1996, Weidenshiling \& Marzari 1996, Lin \& Ida 1997)) and tidal circularization. Recent studies of the planet-planet scattering show good coincidence with the observational eccentricity distribution (Marzari \& Weidenschilling 2002, Chatterjee Ford, \& Rasio 2007, Ford \& Rasio 2007).

A formation probability of the close-in planet (i.e., the pericenter distance becomes small enough and the tidal circularization takes place) is the problem of the slingshot model. In this paper, we investigate the efficiency of the slingshot process in producing 


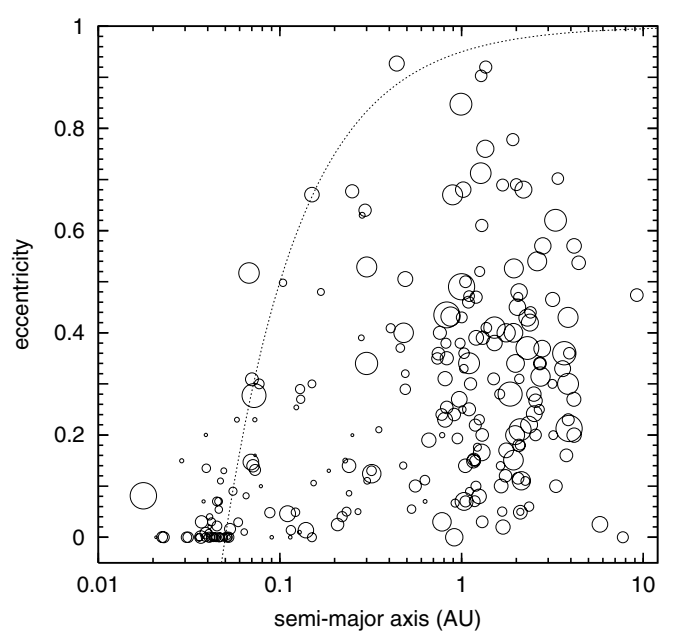

Figure 1. Distribution of orbital eccentricity and semimajor axis of observed extrasolar planets. Circle sizes are proportional to $(m \sin i)^{1 / 3}$. Dotted line shows pericenter distance $q=0.05$ AU.

close-in planets by including a dynamical tide into the simulations. Following to the explanation of numerical models $(\S 2)$, we present the results $(\S 3)$ and give conclution $(\S 4)$.

\section{Methods}

Following to the simulations of the Jumping Jupiter model done by Marzari \& Weidenschilling 2002, we consider a system of three planets with equal mass $\left(m=1 / 1000 m_{\odot}\right)$ orbiting around a solar mass star in circular orbits. Their initial semi-major axes are $a_{1}=5.00, a_{2}=7.25$, and $a_{3}=9.50 \mathrm{AU}$ and their inclinations are $i_{1}=0.5^{\circ}, i_{2}=1.0^{\circ}$, and $i_{3}=1.5^{\circ}$. We use a formula of the change of energy and angular momentum caused by the dynamic tide obtained by Ivanov \& Papaloizou 2004. We change the orbit of planet at the time of its pericenter passage (an impulse approximation) in a same orbital plane (see Nagasawa, Ida, \& Bessho 2008 for details). The tide is a function of mass and radius of planets. We performed 4 set of simulations. The radius of planet are $R=1$ (Cases 2 and 4 ) and $2 R_{\mathrm{J}}$ (Cases 1 and 3 ). The planet does not rotate in Case 1 and Case 2. The change of angular momentum by tidal force is set to zero in Case 3 and Case 4.

Because of the chaotic nature of the scattering processes, we integrate 100 different systems with different orbital angles in each set. Basically, we integrate the orbit for $10^{7}-10^{8}$ yr. We stop the calculation when the change of energy and angular momentum by tidal force overcome the magnitude of orbital energy and angular momentum that the planet has. We also stop the calculation when a planet hits the surface of the host star of 1 Solar Radius.

\section{Results}

A typical evolution of semi-major axes, pericenters, and apocenters of three-planet system in the case that a close-in planet is formed is shown in Figure 2 (Case 4). After several encounter events, an initially outermost planet is scattered into $3 \mathrm{AU}$. At $2.16 \times 10^{6} \mathrm{yr}$, the planet obtains a large inclination $(\sim 1.4$ radian $)$ and eccentricity $(\sim 0.83)$ as a result of an encounter. The inclination is exchanged for the eccentricity by Kozai mechanism. 


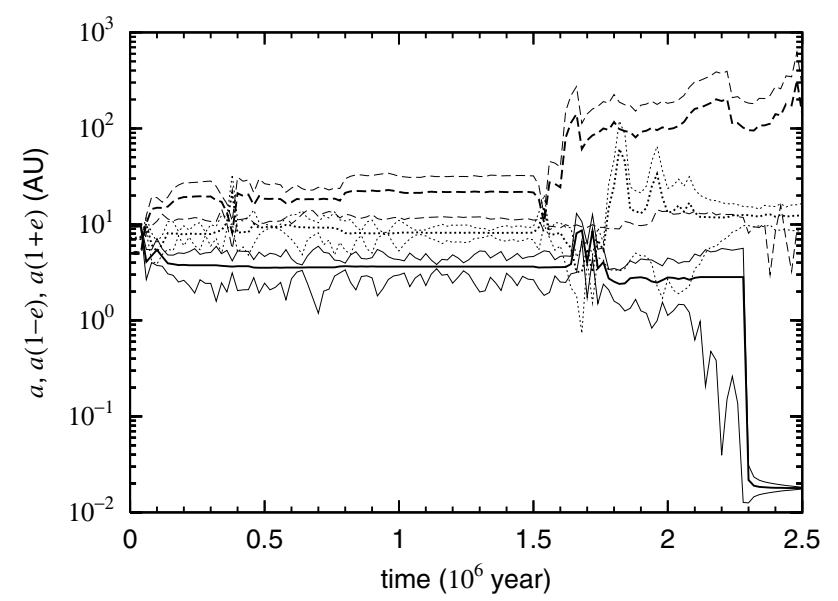

Figure 2. Typical evolution of the semi-major axes of three planets. Thin lines show evolution of pericenters and apocenters.

The pericenter of the planet approaches the star. The semi-major axis decreases immediately in $10^{4}$ years. The damping of the eccentricity takes slightly longer time, but the planet is circularized in $\sim 2 \times 10^{5} \mathrm{yr}$.

A compiled result is shown in Table 1 . There is no significant difference between the models. In total, we find the close-in planet in 33\% cases. On average, a close-in planet is produced during three-planet interaction in $26 \%$ cases. In about $7 \%$ cases, a closein planet is formed after an ejection of one of the planets. The ratio of the later case is consistent with the result of Marzari \& Weidensilling 2002 that takes into account an evolution of longer period. This shows that about $3 / 4$ close-in planets are formed during three-planet interaction stage and not after the stabilization of the system. After one of the planet is scattered inward and detached from other planets, Kozai evolution timescale for the planet can be shorter than the timescale of the planet-planet scattering. The eccentricity and inclination of the innermost planet is changed whenever one of the outer planets approaches. When the planet enters in a good Kozai-state, the pericenter approaches to its host star and the planet becomes the close-in planet.

The final eccentricity and semi-major axis of the formed planet is shown in Figure 3. When the pericenter of the planet does not approach to its host star enough for the tidal circularization, the orbital energy of planet is conserved. In this case, the minimum semi-major axis achievable $\left(a_{\mathrm{min}}\right)$ is determined by the energy conservation, $1 / a_{\mathrm{min}}=$ $1 / a_{1}+1 / a_{2}+1 / a_{3}$. In our initial setting, $a_{\mathrm{min}}=2.25 \mathrm{AU}$. Therefore, the inner planets that

Table 1. Results of numerical simulations

\begin{tabular}{|c|c|c|c|c|c|}
\hline Set & Planet radius $\left[R_{J}\right]$ & ] Rotations & $\mid H J_{3}{ }^{1}$ & $H J_{2}{ }^{2}$ & $\mid N_{\text {star }}{ }^{3}$ \\
\hline Case 1 & 2 & No & | 31 & 7 & 1 \\
\hline Case 2 & 1 & No & 21 & 8 & 1 \\
\hline Case $3 \mid$ & 2 & Yes & 25 & 8 & 2 \\
\hline Case 4 & 1 & Yes & 26 & 6 & 2 \\
\hline
\end{tabular}

\footnotetext{
Notes:

${ }^{1}$ Number of close-in planets produced during a three-planet interaction

${ }^{2}$ Number of close-in planets produced after an ejection of a planet

${ }^{3}$ Number of planets collided to their host star
} 


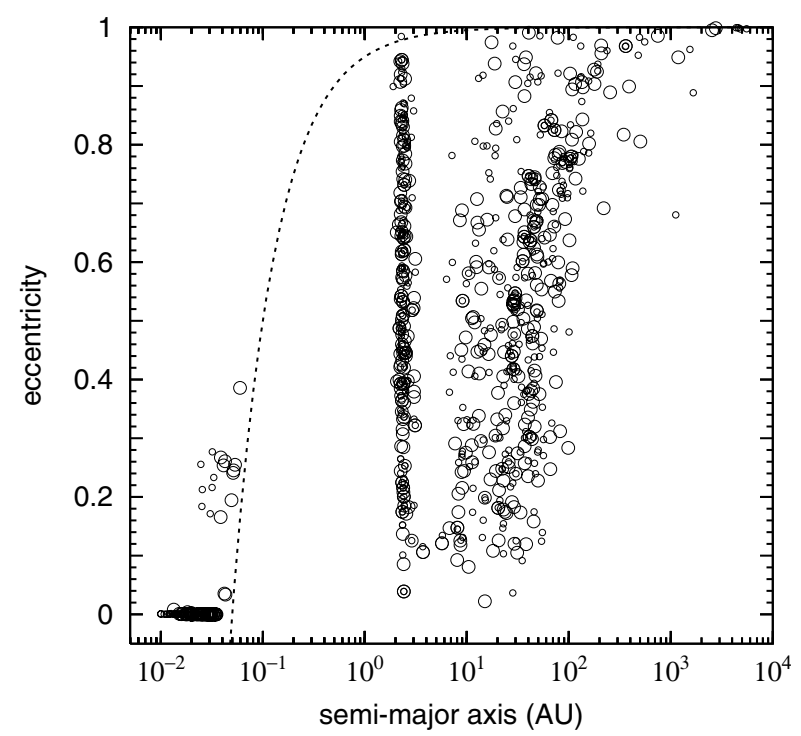

Figure 3. Eccentricities and semi-major axes of formed planets in all simulations at $10^{8}$ year. Planets formed in Case $1 \&$ Case 3 are shown by larger circles. Dotted line shows pericenter distance $q=0.05$ AU.

are not tidally circularized are distributed around 2.5 AU. On the other hand, semi-major axes of outer planets are broadly spread, since a slight change of the orbital energy causes a large difference in the semi-major axis in the outer region. A planet-planet scattering spreads the eccentricities. Although a large number of the formed close-in planet are circularized in $10^{8}$ years, some of the planets are not fully circularized in $10^{8}$ years, and keep moderate eccentricities. This happens in a case that the circularization occurs after an ejection of a planet.

A inclination distribution of planets that are scattered inward in all simulations is shown in Figure 4. Most planets that are not tidally circularized keep relatively small inclinations. On the other hand, the close-in planet (solid line) tends to have a widely spread inclination distribution. Non-negligible fraction of close-in planets has a retrograde rotation $(i>\pi / 2)$ compared to an original orbital plane that is nearly perpendicular to the stellar spin axis. Basically, the Kozai mechanism is effective for planets injected to highly inclined orbits. The tidal circularization starts when the eccentricity becomes high. Therefore, although the close-in planet is formed through Kozai mechanism and has a large inclination at first, the inclination is near the minimum of allowed range when the circularization is started, and the final inclination distribution tends to be spread.

\section{Conclusions}

We investigated the efficiency of the slingshot process - a coupled process of a planetplanet scattering and a tidal circularization - in producing close-in planets. Starting from a system that three Jovian planets are orbiting in unstable circular orbits at $a_{1}=5.00$, $a_{2}=7.25$, and $a_{3}=9.50 \mathrm{AU}$ with small relative inclinations, we calculated their orbital evolution including the effect of dynamical tide. We found that the close-in planets are formed in about $1 / 3$ cases. This high percentage is because that Kozai mechanism works 


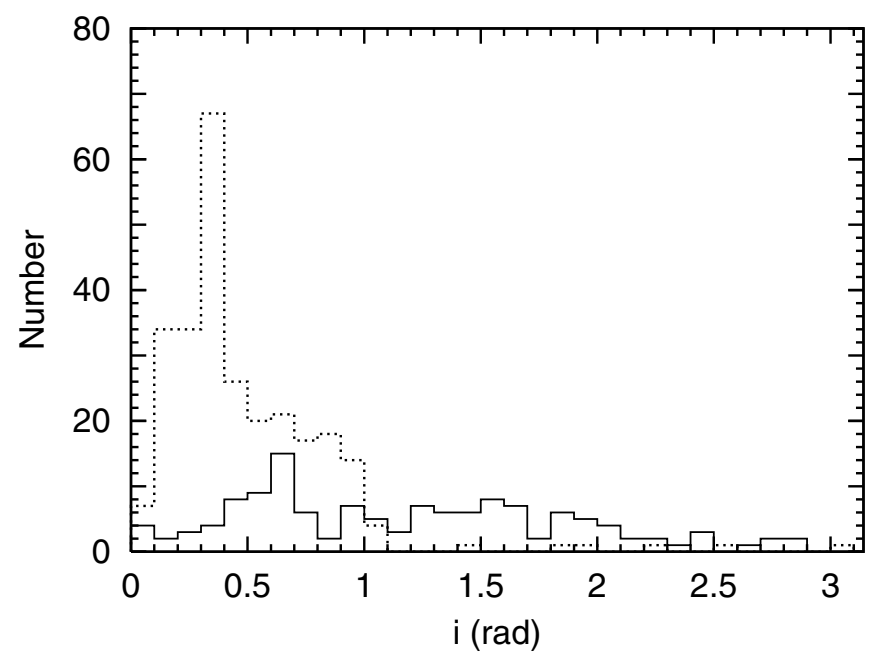

Figure 4. Histogram of inclination of planet scattered inward in all simulations. Solid line shows a number of planets that becomes a close-in planet. Dotted line shows planets that are scattered inward but that are not tidally circularized.

on a inwardly scattered planet and the pericenter of planet approaches the host star enough for tidal circularization.

The inclination of the formed close-in planet is widely spread. Although their tidal lifetime can be short, about a half of the formed close-in planets has retrograde rotations. Observations of Rossiter-McLaughlin effect of 5 transiting close-in planets show that their orbital planes are almost aligned with the stellar spin axis (Narita et al. 2007, Winn et al. 2005, Winn et al. 2006, Winn et al. 2007, Wolf et al. 2007). This may suggest that the slingshot mechanism is not a main process for the formation of close-in planets. The contribution of the slingshot process to the formation of hot planet relative to the Type-II migration will be revealed from more observation of the Rossiter-McLaughlin effect.

\section{Acknowledgments}

This work is supported by MEXT KAKENHI(18740281) Grant-in-Aid for Young Scientists (B) and MEXT's program "Promotion of Environmental Improvement for Independence of Young Researchers" under the Special Coordination Funds for Promoting Science and Technology.

\section{References}

Chatterjee, S., Ford, E. B., \& Rasio, F. A. 2007, ApJ, submitted

Ford, E. B. \& Rasio, F. A. 2007, ApJ, submitted

Ida, S. \& Lin, D. N. C. 2004a, ApJ, 604, 388

Ida, S. \& Lin, D. N. C. 2004b, ApJ, 616, 567

Ivanov, P. B. \& Papaloizou J. C. B. 2004b, MNRAS, 347, 437

Lin, D. N. C. \& Ida, S.1997, Ap. Lett., 477, 781

Marzari, F. \& Weidenschilling, S. J.2002, Icarus, 156, 570

Nagasawa, M., Ida, S., \& Bessho, T. 2008, ApJ, in press

Narita, N., et al. 2007, PASJ, 59, 763

Rasio, F. \& Ford, E. 1996, Science, 274, 954 
Weidenschilling, S. J. \& Marzari, F. 1996, Nature, 384, 619

Winn, J. N., et al. 2005, ApJ, 631, 1215

Winn, J. N., et al. 2006, Ap. Lett., 653, 69

Winn, J. N., et al. 2007, Ap. Lett., 665, L167

Wolf, A. S., Laughlin, G., Henry, G. W., Fischer, D. A. Marcy, G., Butler, G., \& Vogt, S. 2007, ApJ, 667, 549 